\title{
EL TRABAJADOR AUTO CONTROLADO; DE LA TEORÍA A LA PRÁCTICA*
}

\author{
Jairo Omar Delgado Mora ${ }^{1}$
}

Para citar este artículo: Delgado, J. (2015). El trabajador auto controlado; de la teoría a la práctica. Inquietud Empresarial. Vol. XV (2), 115-130.

Fecha de recepción: 24 de julio de 2015 Fecha de aceptación: 10 de noviembre de 2015

\footnotetext{
Artículo de reflexión producto de la necesidad de una organización. Grupo de Investigación GRINDEP

Magíster en Administración. Universidad Pedagógica y Tecnológica de Colombia, jairo.delgado@uptc.edu.co
} 


\title{
Resumen
}

El presente documento, tiene por objeto reflexionar, establecer e implementar una metodología propia que permita favorecer la obtención de las metas organizacionales por parte de los trabajadores del área de filtración de la principal empresa de cervezas del país, enfocando las actividades cotidianas de los mismos hacia la obtención de esas metas.

El soporte teórico que respalda esta metodología se ha construido en el documento presentado por Delgado (2015). De esta manera, éste documento muestra la manera como se desarrolló esta metodología, que permitió obtener los objetivos operacionales planteados.

Palabras claves: poder, realización, afiliación, producto terminado, six sigma

\section{The auto controlled worker; from the theory to the practice}

\begin{abstract}
This document, has as an objective reflect, to establish and implement a methodology to facilitate obtaining the organizational goals made by the workers from the filtration area of the main beer company in the country, focusing on everyday activities towards obtaining the same goals.

The theoretical support behind this methodology, is built on the document presented by Delgado (2015). Hence, this paper shows how this methodology was developed that allowed to obtain the raised operational objectives.
\end{abstract}

Key words: power, performance, affiliation, finished product, six sigma. 


\section{O trabalhador auto controlado; da teoría a prática}

\section{Resumo}

O presente documento, tem por objeto refletir, establecer e implementar uma metodologia própria que permita favorecer a obtenção das metas organizacionais por parte dos trabalhadores da área de filtração da principal empresa de cervejas do país, enfatizando as atividades cotidianas dos mesmos até a obtenção dessas metas.

O suporte teórico que respalda esta metodologia tem-se construido no documento apresentado por Delgado (2015). Desta maneira, este documento mostra a maneira como se desenvolveu esta metodologia, que permitiu obter os objetivos operacionais planejados.

Palabras chave: poder, realização, afiliação, produto terminado, six sigma. 


\section{Introducción}

Con base en el documento, El perfil del trabajador autocontrolado, presentado previamente, Delgado (2015), y como parte del compromiso adquirido en el mismo, a continuación, se pretende hacer de manera sintética la manera como fueron aplicados esos conceptos teóricos en el área de filtración de cervezas de la principal empresa cervecera del país. De igual manera y buscando que los documentos escritos, producto de un trabajo, sean muy breves, concisos, pero sin perder el rigor académico. A continuación se pone en consideración la metodología que permitió que los trabajadores en mención obtengan de manera consistente esas metas planteadas.

La metodología que se pretende implementar se soporta en conceptos presentados por: Liker (2004) acerca de la manera como se resuelven las situaciones problemáticas, los círculos de calidad y el enfoque en los desperdicios. La operacionalidad de los conceptos emitidos por estos autores, fundamentalmente se soporta en los métodos planteados por Ishikawa, a través del análisis de causas basado en la espina de pescado, Deming y el ciclo; PHVA y dentro de estos se hace una inmersión del método ITACONE, Romero y More (2013).

\section{Perfil del trabajador auto controlado}

Realizado el ejercicio preliminar, a través del cual se logra establecer el perfil del trabajador auto controlado, (Figura 1). Surge la necesidad de determinar las oportunidades de mejora que tiene los trabajadores, objeto de evaluación.

Esas oportunidades de mejora en las competencias de los trabajadores, se vuelven visibles, si se hace una revisión de las 11 principales debilidades competitivas planteadas por Solano en su documento en referencia Solano, Bravo y Giraldo (2012):

- Debilidades en el direccionamiento estratégico de la fabricación.

- Baja capacidad administrativa para vincularse con el mercado internacional.

- Sistemas de producción no alineados con las prioridades competitivas.

- Ausencia de sistemas integrados de planeación, programación y control de la producción.

- Deficiencias en los sistemas de costos de producción.

- Desconocimiento de la capacidad de producción.

- Deficiencias en los sistemas de calidad. 
Figura 1. Perfil del trabajador auto controlado

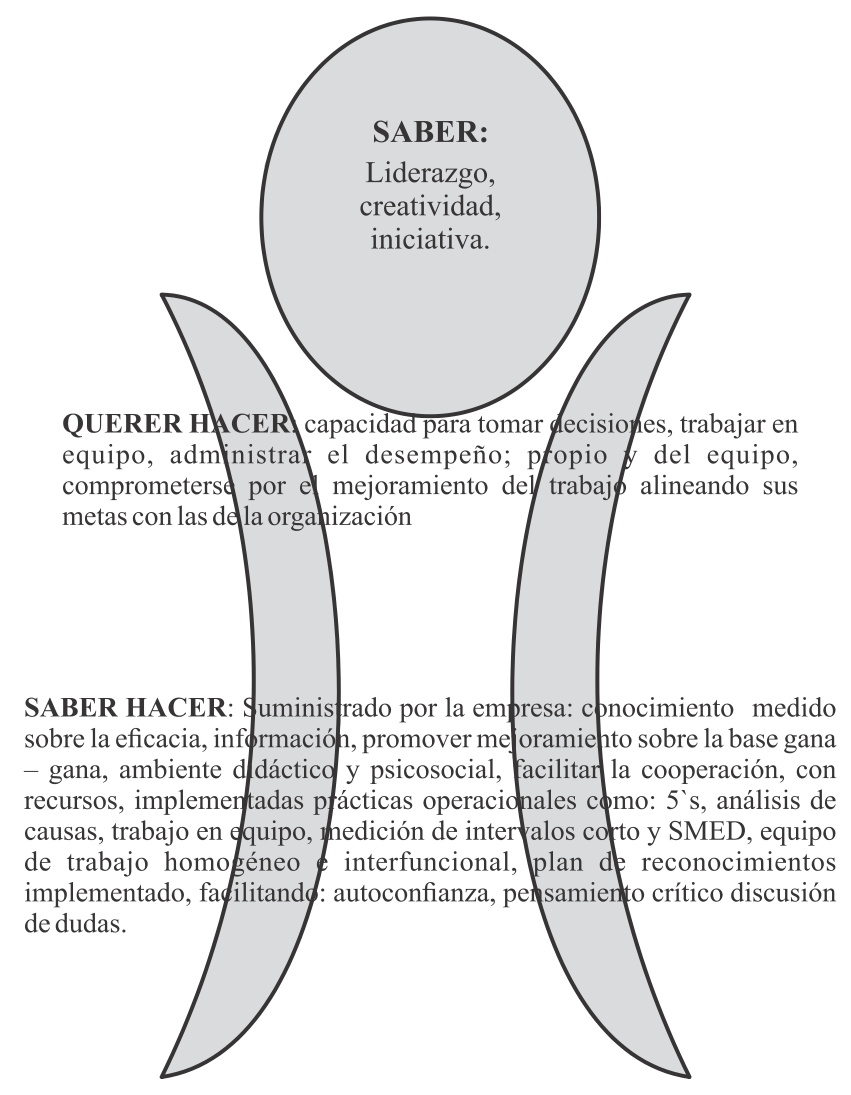

Fuente: Delgado (2015, p. 132)

- Atraso y obsolescencia tecnológica.

- Dificultad para incursionar en mercados sofisticados con productos más diferenciados.

- Falta de una mejor comprensión de sus fortalezas competitivas y debilidades, contemplado también por Miranda (mencionado en Rosales et al., 2015).

- Falta una actitud más agresiva hacia el aprendizaje y la modernidad institucional.

Con base en esas debilidades competitivas dentro de una organización, se establece que es necesario hacer una identificación de los valores que involucran los aspectos que aparecen en la figura 2. 
Figura 2. Debilidades competitivas

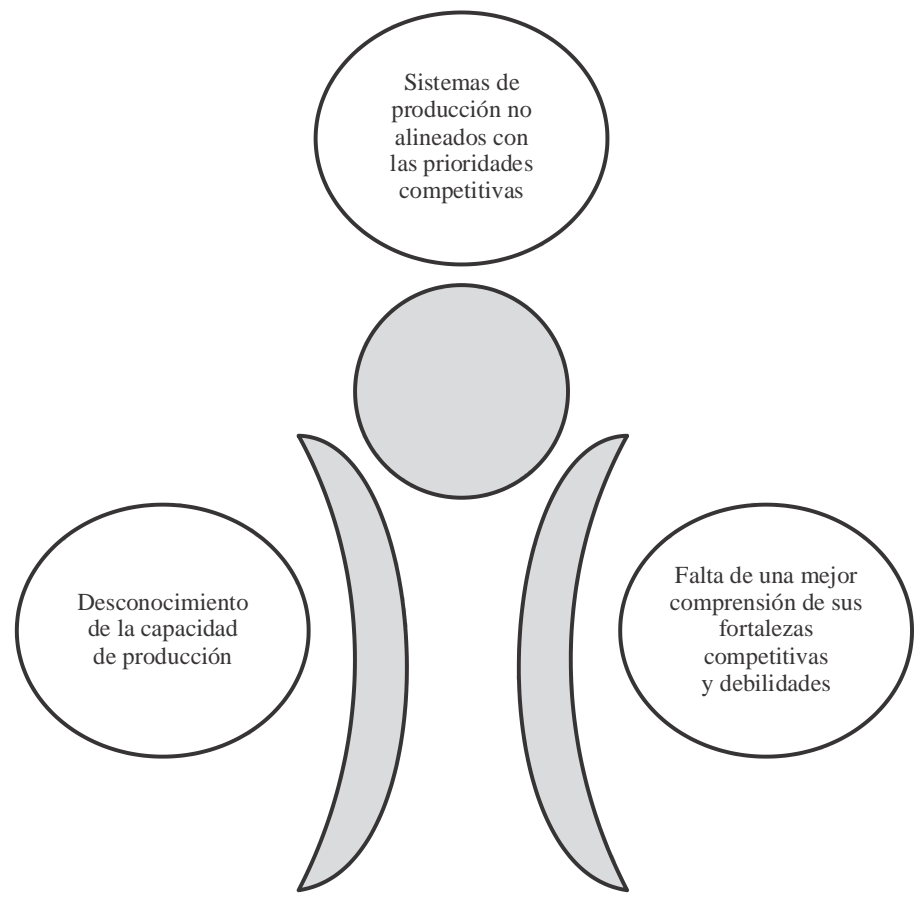

Fuente: elaboración propia

Con base en las competencias definidas en la figura $1 \mathrm{y}$ las debilidades competitivas determinadas en la figura 2, se hace una revisión de las competencias establecidas por el área de talento humano de la cervecera y cuyas bases se han colocado en las figuras 3 y 4 . 
Figura 3. Modelo de competencias para los trabajadores de la cervecería
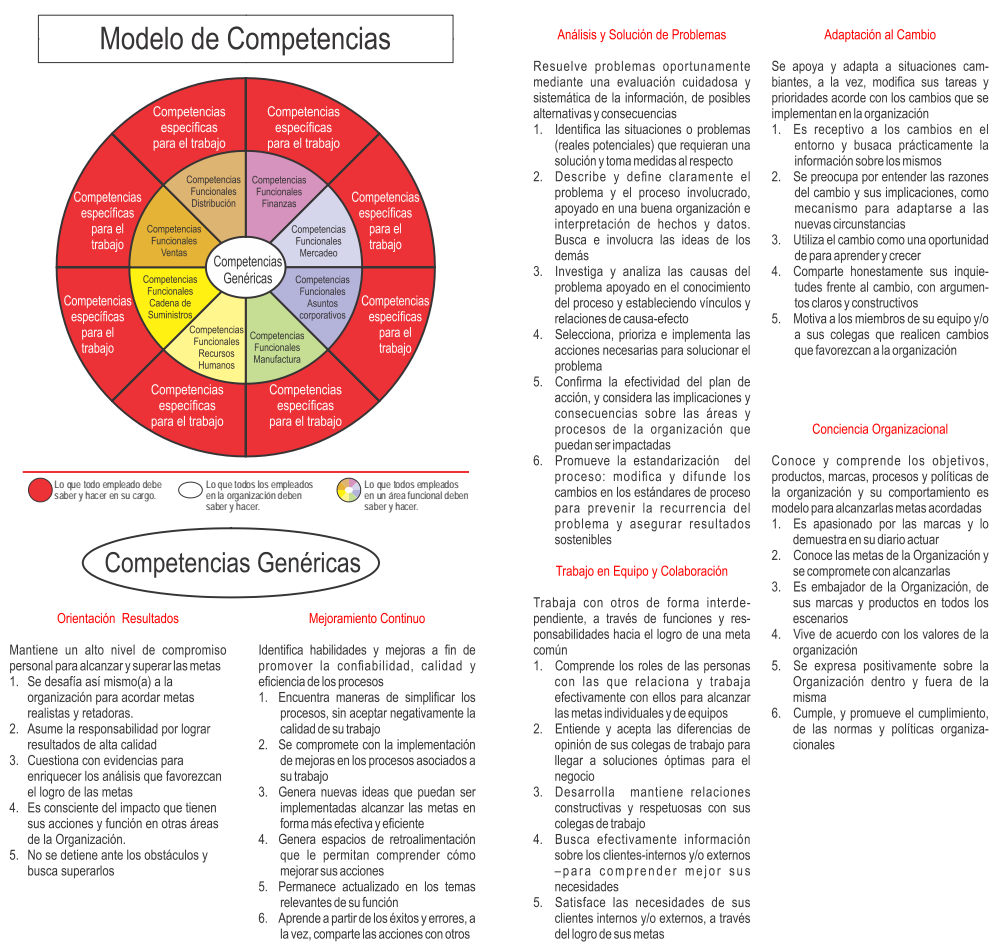

Fuente: Competencias (2009). Portal corporativo de BAVARIA S.A.

Figura 4. Etapas evolutivas del conocimiento técnico.

\begin{tabular}{|c|c|c|c|}
\hline \multicolumn{4}{|c|}{$\begin{array}{l}\text { Demuestra conocimiento técnico } \\
\text { Desarrolla el conocimiento necesario para comprender y traducir los objetivos estratégicos a ser } \\
\text { implementados por su área y otras del negocio. Busca la mejor forma de realizar su trabajo, bajo los } \\
\text { principios de la ética, la autogestión y autocontrol, teniendo en cuenta las normas/reglas. }\end{array}$} \\
\hline Etapa 1 & Etapa 2 & Etapa 3 & Etapa 4 \\
\hline $\begin{array}{l}\text { Aplica conocimiento } \\
\text { básico } \\
\text { técnicolfuncional para } \\
\text { complementar el trabajo. }\end{array}$ & $\begin{array}{l}\text { Utiliza la experticia } \\
\text { técnica para aconsejar } \\
\text { a los demás, } \\
\text { aumentando la } \\
\text { percepción de ser una } \\
\text { persona en la que se } \\
\text { puede confiar y añade } \\
\text { valor a las decisiones } \\
\text { de sus clientes. }\end{array}$ & $\begin{array}{l}\text { Identifica los aspectos } \\
\text { que deben tener un } \\
\text { seguimiento, ser } \\
\text { evaluados y/o } \\
\text { controlados, para } \\
\text { aplicar los estándares } \\
\text { normativos del proceso } \\
\text { a su cargo y de las } \\
\text { áreas relacionadas. }\end{array}$ & $\begin{array}{l}\text { Resuelve las } \\
\text { ambigüedades que } \\
\text { puedan presentarse en } \\
\text { el análisis y evaluación } \\
\text { de los resultados } \\
\text { financieros y/o de } \\
\text { tecnología de la } \\
\text { información para } \\
\text { cumplir con los } \\
\text { objetivos estratégicos } \\
\text { del negocio. }\end{array}$ \\
\hline
\end{tabular}

Fuente: Competencias (2009) Portal corporativo de BAVARIA S.A 
En las figuras enunciadas anteriormente, el área de talento humano ha plasmado las competencias genéricas (conocimiento), dentro de las cuales se mencionan: orientación a resultados, mejoramiento continuo, análisis y solución de problemas, trabajo en equipo, adaptación al cambio y conciencia organizacional. Igualmente, se mencionan las competencias funcionales (ventas, manufactura y mercadeo) y específicas de los diferentes cargos; las cuales se han alineado con el proceso de manufactura de clase mundial a través de las prácticas de: trabajo en equipo, mejora enfocada, liderazgo y gestión del cambio, flexibilidad de la producción y medición visual del desempeño.

Un diagnóstico realizado por el área técnica de manufactura, con relación a los temas de competencia contemplados en las figuras 2,3 y 4, establece que los trabajadores cuentan con un alto compromiso laboral, experiencia técnica y muy buenas bases en el concepto de calidad. Sin embargo, consideran que es necesario implementar prácticas operacionales estructuradas, empoderar a los trabajadores con claridad en sus actividades a desempeñar y sus responsabilidades, identificando y eliminando tareas que no agregan valor. Vicepresidencia Técnica (2009).

Con el anterior diagnóstico realizado, las debilidades competitivas "Falta de una mejor comprensión de sus fortalezas competitivas y debilidades" y "sistemas de producción no alineados con las prioridades competitivas", mostradas en la figura 2, fueron superadas.

El tema asociado con "desconocimiento de la capacidad de producción”, tiene una relación directa con una herramienta de estadística básica y es la capacidad de proceso en operación a través de la determinación de valores como el $\mathrm{C}_{\mathrm{P}} \mathrm{y} / \mathrm{o} \mathrm{CP}_{\mathrm{k}}$, o mejor aún con el concepto de "six sigma".

\section{Six Sigma}

Six sigma se ha planteado como "un vehículo para un cambio estratégico... un enfoque organizacional para alcanzar la excelencia”. Sociedad Colombiana de Six Sigma (2008). Sigma es una letra griega que significa una unidad estadística de medición, usada para definir la desviación estándar de una población, esta mide la variabilidad o dispersión de un conjunto de datos. Six sigma es entonces una medición de variabilidad. Es un nombre que se le ha dado para identificar cuántos de esos datos están dentro de los requerimientos de los clientes, a mayor sigma de un proceso, la mayor salida de éste, ya sea un producto o un servicio, cumplirá con los requerimientos del cliente o sea, menor número de defectos. 
A continuación se describe la metodología seguida para intentar el acercamiento hacia estos objetivos.

\section{Metodología}

Para satisfacer los objetivos definidos en el presente trabajo, se planteó el siguiente diseño metodológico de tipo cualitativo que surgió de las necesidades de la organización dentro de las cuales está el alcance del indicador sigma de proceso y de las expectativas planteadas por los gerentes del área técnica con relación al desempeño de los trabajadores. De esta manera, se parte de la recolección de información obtenida del proceso de elaboración de cerveza/filtración en evaluación, que se ha digitado en archivos magnéticos por los trabajadores en cuestión. Desde aquí, se evalúa la manera como los trabajadores interpretan y soportan sus actividades cotidianas en procura de mejorar, los indicadores de los procesos a su cargo de donde surgen una serie de aspectos convergentes entre la opinión de los trabajadores y la gerencia del área técnica, como la necesidad de autocontrol, que finalmente se plasma en una herramienta que va a permitir satisfacer los objetivos planteados en el presente trabajo.

\subsection{Justificación}

El concepto de autocontrol es relevante en la medida en que sea aplicado en las actividades cotidianas de una organización.

\subsection{Problemática}

Una vez que se ha respondido a la pregunta ¿cuáles son las características que deben cumplir los trabajadores enfocados en el autocontrol?, en el documento de Delgado (2015), surge la necesidad de establecer la manera como se pueden hacer prácticas estas características del trabajador controlado.

De esta manera, a continuación se hace una revisión de la manera como se logró convertir esta necesidad organizacional en algo práctico.

\subsection{De la teoría a la práctica}

Como se mencionó anteriormente, los parámetros del proceso a medir se definen por la alta dirección. Sin embargo, los trabajadores requieren de la apropiación y de una herramienta metodológica que permita promover el mejoramiento de esos resultados. 
Haciendo una revisión del modelo planteado por la multinacional que opera en Colombia para producir cervezas, se ha encontrado que este modelo proviene de los ocho principios establecidos por John Kotter, (mencionado en manufactura de clase mundial Boyacá plan, 2011). De esta manera, en la figura 7, se ha construido un cuadro que resume la articulación entre los principios que pretende implementar la alta gerencia de la multinacional, su fuente obtenida de los principios de Kotter y el "manufacturing way", que es el instrumento en proceso de implementación en las cervecerías.

Figura 5. Instrumento de implementación de las mejores prácticas operacionales.

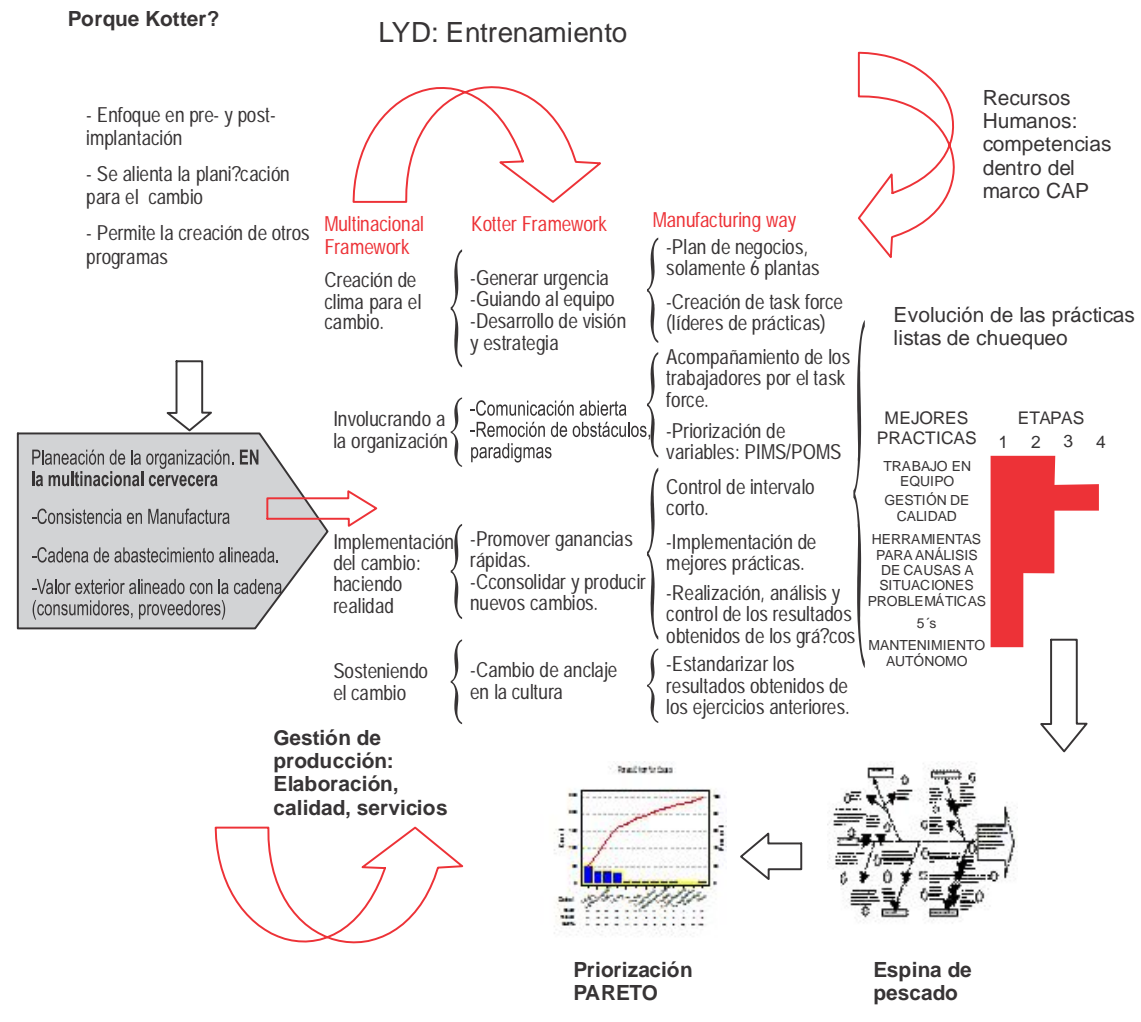

Fuente: elaboración propia

En la figura 5, se observa que la evolución de las prácticas y, específicamente la que tiene que ver con el área de calidad, se encuentra su evolución entre las etapas 3 y 4, figura 4. 
De esta manera, es necesario generar una herramienta que permita conseguir que se supere el "Desconocimiento de la capacidad de producción", figura 2, pero que a su vez se articule con la necesidad de autocontrol sobre la base de la aplicación de otras mejores prácticas, como lo establece el perfil del trabajador auto controlado y es una necesidad detectada por el área técnica, De igual manera, se espera que esta herramienta potencie la práctica de calidad que es una gran fortaleza.

Con base en la figura 5, el siguiente paso es buscar cerrar la brecha entre el valor ideal de las metas y su estado "actual".

Para ello, el presente trabajo se apoya en la metodología establecida por el modelo ITACONE, Romero y More (2013), Bribiescas (2009), Goldratt (2008), y el documento de Bloom et al. (2012) cuyas etapas de realización se han conceptualizado en la figura 6.

Figura 6. Metodología para resolver situaciones problemáticas

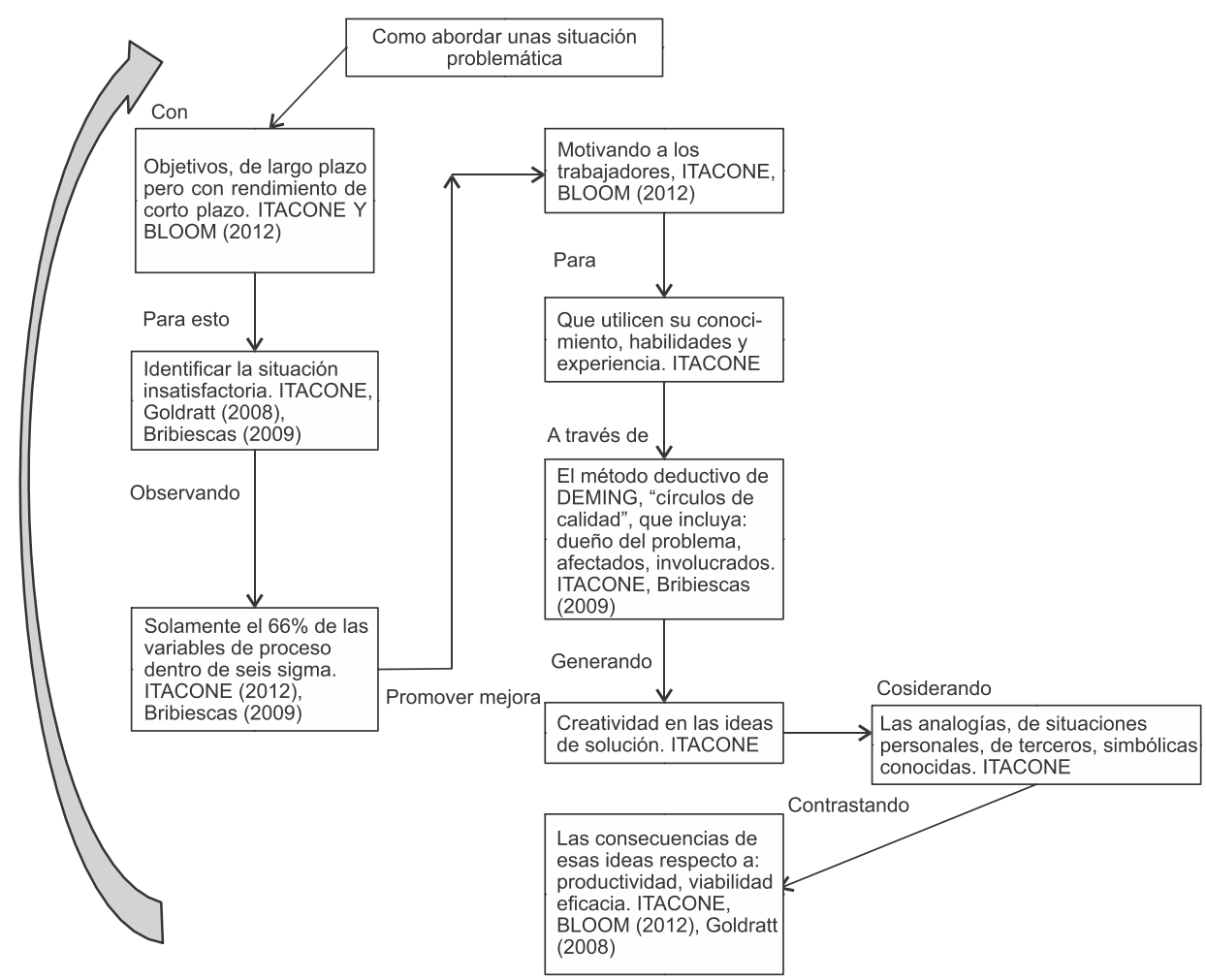

Fuente: elaboración propia. 
Considerando las primeras 3 etapas de la figura 6 y después de verificar el estado de avance de la práctica de calidad en la figura 5, a continuación se presenta el diagrama de análisis de causas para poder colocar el $88 \%$ de los parámetros fisicoquímicos de las variables de proceso dentro de 4.5 sigmas de proceso.

Con base en lo expuesto a lo largo de este trabajo y revisando el desempeño de los 17 aspectos contemplados en la figura 7 , se considera que los puntos que aún están pendientes por satisfacer son:

- ¿El trabajador monitorea su desempeño?

- ¿Se ha establecido alguna frecuencia para las reuniones de equipos para la solución de problemas?

- ¿Se aplican las herramientas para análisis de causas: quick fix, 3 preguntas básicas 5 ¿por qué?

- ¿El trabajador identifica cuando un producto está fuera de "defectos"? (valores dentro de especificaciones, sigma de proceso). ¿Sabe el impacto de disminuir la variabilidad?

- ¿El trabajador tiene la capacidad y se le ha permitido tomar decisiones de operación y corrección del proceso?

- ¿Existe un plan de reconocimientos para los equipos de trabajo, hay buen trato a los trabajadores y un buen ambiente laboral? 
EL TRABAJADOR AUTO CONTROLADO; DE LA TEORÍA A LA PRÁCTICA

Figura 7. Causas que generan brecha en la consecución de las metas

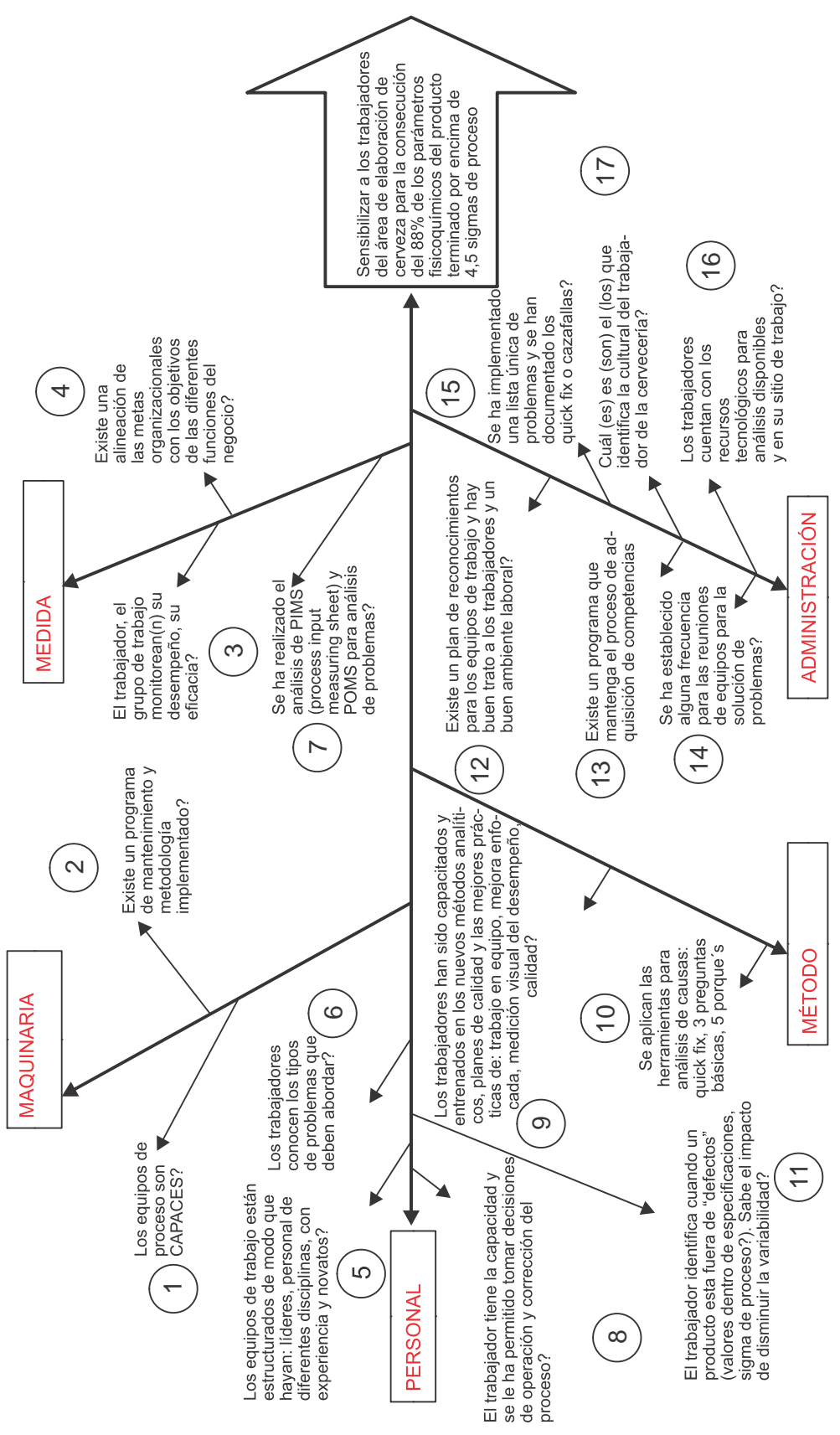

Fuente: elaboración propia 


\section{Conclusiones}

Tomando como base el diagrama de la figura 6 y, la metodología planteada en este documento para resolver situaciones problemáticas, figura 7. Se obtuvieron las anteriores preguntas, que son el objeto final del trabajo y por ende corresponden a las conclusiones del mismo.

En la figura 8, se ha colocado a manera de ejemplo, una de las variables que fueron monitoreadas por parte de los trabajadores. Por supuesto que para la implementación de esta herramienta estadística fue necesaria la sensibilización y entrenamiento del personal operativo, no solamente en aspectos básicos de control, sino en una serie de prácticas operacionales como: trabajo en equipo, enfoque en problemas gerencia visual, cuyo soporte teórico y monitoreo se muestra en la figura 5.

El monitoreo de las variables de proceso son diligenciados por los diferentes equipos de trabajo conformados interdisciplinarmente entre los mismos trabajadores y sobre la desviación presentada tomaron decisiones que ellos implementaron.

Figura 8. Monitoreo y ajuste en tiempo real de las variables de proceso

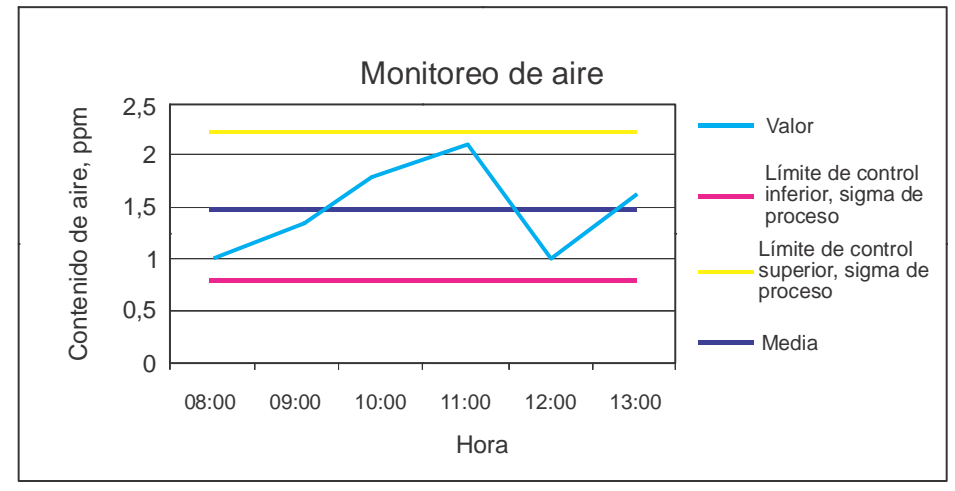

Fuente: Elaboración propia

Así mismo y con base en el diagrama de la figura 7, se realizó un análisis con base en lo definido por Deming, las reuniones de retroalimentación sobre el día a día se hacen cada cambio de turno, toman 12 minutos, y de allí puede surgir la necesidad de utilizar alguna herramienta estructurada para el análisis de causas a problemas presentados como; las 3 preguntas, 5 por qué o la configuración del diagrama de Ishikawa. 
La respuesta a la última pregunta y cuyo resultado obedece a la metodología aquí plasmada para la consecución de los resultados, se muestra a partir del indicador de efectividad organizacional, el cual es medido por una entidad independiente de la organización y cuyo resultado fue del $90.5 \%$ de satisfacción por parte de los trabajadores.

La implementación de esta metodología ha favorecido que el valor de las variables de proceso y dentro de 4.5 sigmas, hayan pasado en 8 meses del $66 \%$ al $88.1 \%$ de valores entre especificaciones.

Finalmente, todo este esfuerzo ha permitido que la cervecería, se encuentre ubicada en el primer lugar, sostenidamente desde hace más de 2 años, entre más de 130 plantas en el mundo.

\section{Referencias Bibliográficas}

Bribiescas, F. (2009). Modelo para la formulación y despliegue de estrategias de manufactura. Revista Internacional Administración y Finanzas. 2(1), 93-109.

Bloom, N., Sadun, R., y Reenen, J. V. (2012). Does management really work?: How three essential practices can address even the most complex global problems? Harvard Business Review: HBR, 90(11), 76-82.

Competencias (2009). Desarrollar el personal. Vicepresidencia de recursos humanos. Bogotá Colombia: portal corporativo de BAVARIA S.A. Recuperado de: http:/ /bavaria/C1/GD/default.aspx

Delgado, J. (2015). El perfil del trabajador autocontrolado. Revista Inquietud Empresarial. Vol. XV (1), 78-103.

Goldratt, E. (2008). La meta, un proceso de mejora continuo. 3ra Ed. Argentina: Ed. Granica S.A.

Liker J. (2004). The Toyota way: 14 management principles from the world's Greatest manufacturer. Estados Unidos: McGraw Hill.

Manufactura de clase mundial Boyacá plan (2011). The Sabmiller Manufacturing way (Los doce factores de éxito para el cambio). Presentación realizada a los gerentes de MCM de BAVARIA, Junio, Bogotá. 
Romero, J. y More, R (2013). Sistema de solución creativa para problemas recurrentes - Itacone. Revista de Ingeniería y Competitividad. Vol.15 (1), 21-35.

Rosales, F., Sánchez, J., Vergara, J. y Pimentel, O. (2015). La disciplina operativa y la microempresa. Revista Inquietud Empresarial. Vol. XV (1), 78-103.

Sociedad Colombiana de Six Sigma (2008). Análisis estadístico. Trabajo presentado en la capacitación sobre seis sigma. Junio de 2010. Duitama / Boyacá.

Solano, M., Bravo, J. y Giraldo. (2012). Metodología de mejoramiento en el desempeño de sistemas de producción. Aplicaciones en Pymes de la confección. Revista de Ingeniería y Competitividad. Volumen 14 (2), 37-52.

Vicepresidencia Técnica (2009). Proyecto de empoderamiento. Presentación realizada a gerentes de BAVARIA. Abril, Bogotá. 